\title{
A Dual-Frequency Quasi-Optical Output System for a THz Gyro-Multiplier
}

\author{
Xiang Li, Xiaoming Liu, Yasir Alfadhl, Kevin Ronald, Wenlong He, Adrian Cross, and Xiaodong Chen
}

\begin{abstract}
This paper presents the investigation of a quasioptical output system for a fourth-harmonic gyro-multiplier in the THz band through geometric optic analysis and three-dimensional full-wave simulation. The output system of the gyro-multiplier needs not only to transfer two high-order waveguide modes into the quasi-Gaussian beams, but also to separate the two beams. A dual-frequency quasi-optical mode converter (DQMC) and a frequency selective surface (FSS) are employed to achieve both goals within this system. Our study has shown that the optimal design of the DQMC requires a special mode selection in the gyro-multiplier. The high-pass FSS is designed, fabricated, and experimentally verified by a THz-TDS system. We have also demonstrated that the designed FSS is capable of handling the high-power output from the gyro-multiplier.
\end{abstract}

Index Terms-Frequency selective surface (FSS), gyromultiplier, output systems, quasi-optical mode converter.

\section{INTRODUCTION}

A GYRO-MULTIPLIER is a high-power THz signal generator based on the combination of traditional gyrotron-like beam-wave interaction [1] and frequency multiplication [2]. Although the operation of the gyro-multiplier has been studied extensively in theory [3], [4] and demonstrated successfully in experiments [5], [6] in recent years, the study of a proper output system for such device has not been reported in the literature. Unlike the traditional gyrotrons of single-mode operation, the output of a gyro-multiplier contains two modes with one operating at the fundamental electron cyclotron resonance and the other being excited at one of the high-order harmonics. For example, the fourth-harmonic gyro-multiplier with sectioned cavity [2], [6] generates the output waves at 342 and $1368 \mathrm{GHz}$, respectively, as summarized in Table I. A proper output system for the gyro-multiplier should be able not only to convert the

Manuscript received March 15, 2016; revised May 16, 2016; accepted June 13, 2016. This work was supported in part by the National Nature Science Foundation of China under Contract 61401031 and by the Scientific Research Foundation for the Returned Overseas Chinese Scholars, State Education Ministry.

X. Li, Y. Alfadhl, and X. Chen are with the School of Electronic Engineering and Computer Science, Queen Mary University of London, London E1 4NS, U.K. (e-mail: xiang.x.li@qmul.ac.uk; yasir.alfadhl@qmul.ac.uk; xiaodong.chen@qmul.ac.uk).

$\mathrm{X}$. Liu is with the School of Electronic Engineering, Beijing University of Posts and Telecommunications, Beijing 100876, China (e-mail: xiaoming liu@bupt.edu.cn).

K. Ronald, W. He, and A. Cross are with the Department of Physics, Scottish Universities Physics Alliance, University of Strathclyde, Glasgow G4 0NG, U.K. (e-mail: k.ronald@strath.ac.uk; w.he@strath.ac. uk; a.w.cross@strath.ac.uk).
TABLE I

SUMMARY OF THE OUTPUT WAVE CONTENTS IN THE FOURTH-HARMONIC GYRO-MULTIPLIER

\begin{tabular}{lcc}
\hline \hline & $L F$ & $H F$ \\
\hline$s$ & 1 & 4 \\
$f(\mathrm{GHz})$ & 342 & 1368 \\
$P_{0}(\mathrm{~kW})$ & 2 & 0.12 \\
Main Mode & $\mathrm{TE}_{1,3}$ & $\mathrm{TE}_{4,9}$ \\
\hline \hline
\end{tabular}

LF and HF are short for low-frequency and highfrequency, respectively. $s$ is the harmonic number. $f$ denotes the radiation frequency. $P_{0}$ represents the output power.

generated wave to quasi-Gaussian beams, but also to separate the two modes in space.

To transform as well as separate the wave beams, we have to integrate a dual-frequency quasi-optical mode converter (DQMC) and a frequency selective surface (FSS) in the output system. The traditional quasi-optical mode converter (QMC) has been successfully utilized in gyrotrons for the conversion of the single-mode output into quasi-Gaussian beam [7]. It has also been optimized for the frequency tunable gyrotrons operating with a number of neighboring modes at the fundamental harmonic [8]. In the case of the gyro-multiplier, however, the fundamental and harmonic modes are distantly separated in the spectrum. The traditional geometrical optics analysis needs to be modified in the design of a DQMC by taking into consideration the propagation properties of both modes to enable the simultaneous transformation of both contents into quasi-Gaussian beams.

For the separation of the two frequencies, we have employed an FSS. The FSS has been widely used in the low-power range as an effective filter [9]. It has also been shown as operable with high-power microwave radiation [10]. Nevertheless, its operation with high-power $\mathrm{THz}$ radiation has not been considered elsewhere. Here, a high-pass FSS formed by periodic circular perforations on a metal plate is designed, fabricated, and tested.

The remaining part of this paper is organized as follows: Section II discusses the configuration of the system. Section III presents the theory and three-dimensional (3-D) fullwave verification of the DQMC in simulation. Section IV shows the design, fabrication, and testing results of the high-pass FSS of the slot type, with consideration of the high-power incidence. Section V concludes this paper and suggests the future work. 


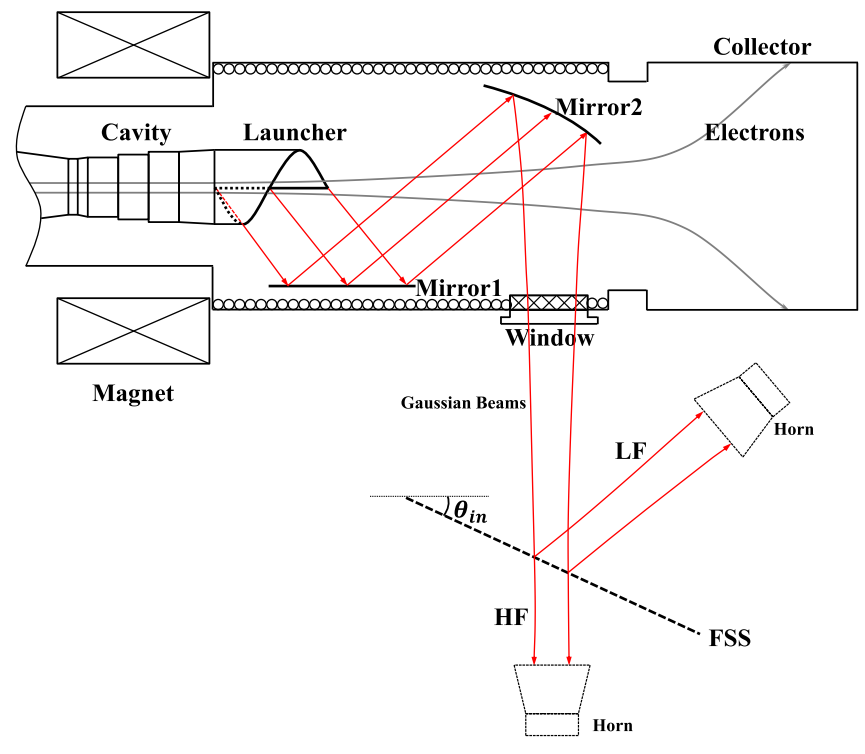

Fig. 1. Overview of the designed output system for the gyro-multiplier.

\section{SySTEM CONFIGURATION}

Since the FSS is a periodic structure, its performance can only be predictable, manipulated, and optimized when operating with the plane waves or the Gaussian beams. Thus, the high-order waveguide modes from the gyro-multiplier should be transformed into quasi-Gaussian beams, before being separated by the FSS.

Fig. 1 shows the schematic view of the designed output system for the gyro-multiplier. A DQMC consisting of a launcher and two mirrors is connected to the output end of the beam-wave interaction cavity. The red lines represent the path of the converted wave beams. Apart from enabling the effective operation of the subsequent FSS, the quasi-optical system is a favorable choice to reduce the transmission and Ohmic loss in the $\mathrm{THz}$ frequency range.

At the waists of the converted quasi-Gaussian beams, an FSS is applied to separate the two frequencies. To avoid reflecting the wave back into the beam-wave interaction system, the FSS is designed for oblique incidence with an incident angle of $\theta_{\text {in }}$. In Fig. 1, the low-frequency content is reflected, while the highfrequency counterpart is transmitted.

\section{DuAL-Frequency QuASI-OPTICAL MOde CONVERTER}

A QMC is usually composed of a launcher to directionally radiate the high-order waveguide modes into free space and several subsequent reflectors to adjust the generated wave beams and form quasi-Gaussian field distributions. The original Vlasov launcher with the regular equation-based mirrors was proposed and demonstrated in 1975 [7]. Since then, several improved versions of the Vlasov launcher and the standard mirrors have been reported in the literature [11]-[18].

However, these improved versions tend to add additional complexity in the design, fabrication, and alignment process when operating at 342 and $1368 \mathrm{GHz}$. Besides, as the THz output power of the gyro-multiplier is relatively low compared with

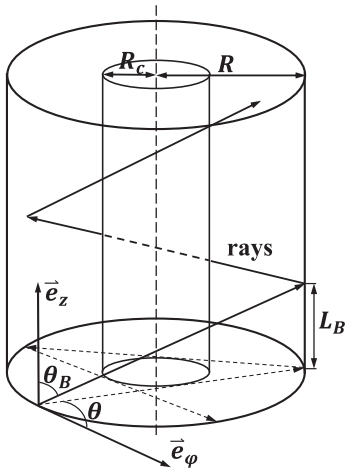

(a)

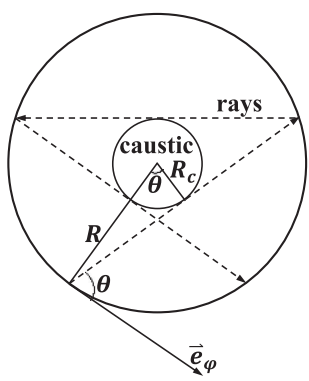

(b)
Fig. 2. Geometrical optics of the mode propagation in a cylindrical waveguide. (a) Side view. (b) Top view.

the traditional gyrotrons, the diffraction and relatively low efficiency of the Vlasov launcher are tolerable. Thus, the Vlasovtype launcher and the standard mirrors are chosen in the designed DQMC for this proof-of-principle study.

According to the geometric optics, the rotating traveling wave in the cylindrical waveguide can be decomposed into a continuous flow of rays, as shown in Fig. 2 [13]. The rays form an angle $\theta_{B}$ with the axis of the waveguide and

$$
\theta_{B}=\arcsin \left(\frac{\mu_{m, n}^{\prime}}{R \cdot k_{0}}\right)
$$

in which $\mu_{m, n}^{\prime}$ is the eigenvalue of the $T E_{m, n}$ mode in a cylindrical waveguide. $m$ and $n$ represent the azimuthal and radial index of the mode, respectively. $R$ is the radius of the waveguide. $k_{0}$ is the wave number of the mode and $k_{0}=2 \pi f / c . c$ is the speed of light in free space. $R_{c}$ is the caustic radius of the modes and $R_{c}=R \cdot m / \mu_{m, n}^{\prime}$. The projections of the rays form an angle $\theta$ [7] with $e_{\varphi}$, which is the unit vector in the $\varphi$ direction of the cylindrical coordinate system. It can be derived that

$$
\theta=\arccos \left(\frac{m}{\mu_{m, n}^{\prime}}\right) \text {. }
$$

The reflecting points of individual ray on the waveguide wall form a helical line, which has an inclination angle $\Phi$, regarding the axial direction, and

$$
\Phi=\operatorname{arccot}\left(\frac{\sin \theta \cdot \cot \theta_{B}}{\theta}\right) .
$$

The distance that a ray has propagated in the axial direction, when it completes a full azimuthal turn, can be obtained as [13]

$$
L=2 \pi R^{2} \frac{k_{z}}{\mu_{m, n}^{\prime}} \sqrt{1-\left(\frac{m}{\mu_{m, n}^{\prime}}\right)^{2}}\left(\arccos \left(\frac{m}{\mu_{m, n}^{\prime}}\right)\right)^{-1}
$$

where $k_{z}$ is the axial wave number of the waveguide mode and

$$
k_{z}=\sqrt{k_{0}^{2}-\left(\frac{\mu_{m, n}^{\prime}}{R}\right)^{2}} .
$$

Considering the rotational property of the rays' propagation inside the cylindrical waveguide, there will be a series of Bril- 


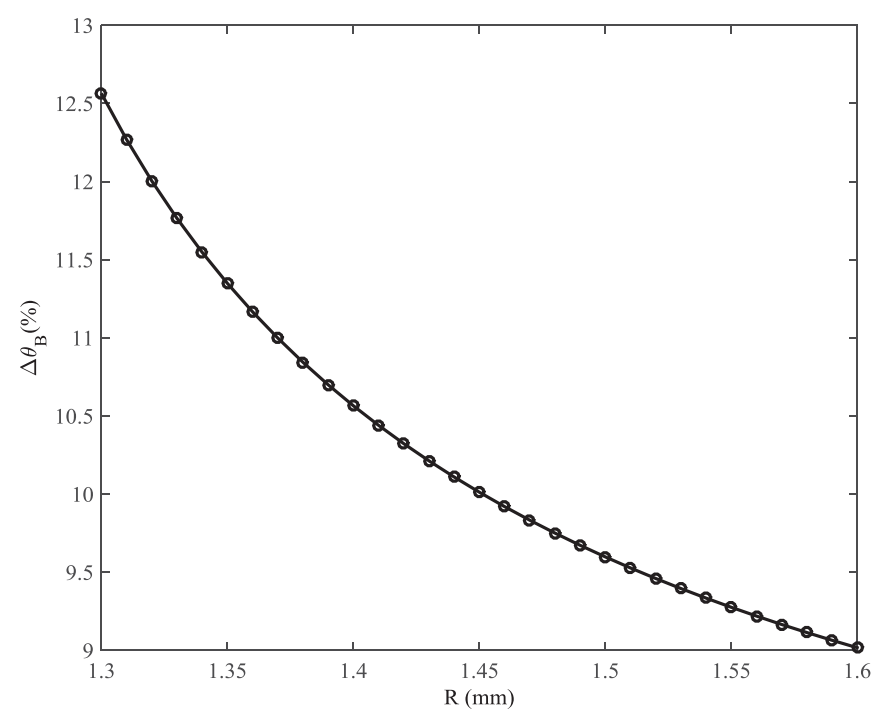

Fig. 3. Variation of $\Delta \theta_{B}$ with the radius of the Vlasov launcher.

louin regions [13] on the internal surface of the waveguide, each of which reflects all the rays exactly once. The axial length of the Brillouin region is $L$ and the azimuthal length $L_{a}$ can be expressed as

$$
L_{a}=2 \theta R .
$$

The aperture of a Vlasov launcher can be defined as the last Brillouin region with length $L$ and width $L_{a}$ located at the end of the launcher.

For the dual-frequency operation, a set of quadratic mirrors is used to guide the two RF beams so the difference of the values of the angle $\theta_{B}$ should be as small as possible, and the Vlasov launcher should be designed to launch both modes effectively.

On the one hand, the difference between the values of $\theta_{B}$ for the two modes can be defined as

$$
\Delta \theta_{B}=\frac{\left|\theta_{\mathrm{BLF}}-\theta_{\mathrm{BHF}}\right|}{\theta_{\mathrm{BHF}}} \times 100 \%
$$

in which $\theta_{\mathrm{BLF}}$ and $\theta_{\mathrm{BHF}}$ are calculated from (1) for the $T E_{1,3}$ and $T E_{4,9}$ modes, respectively. By the combination of (1) and (7), the variation of $\Delta \theta_{B}$ with $R$ is shown in Fig. 3 .

It can be seen that $\Delta \theta_{B}$, while being relatively small, decreases with the increase of $R$. Thus, the choice of $R$ is based on a tradeoff. In order to maintain a relatively small value of $\Delta \theta_{B}$, the value of $R$ should be as large as possible. As the upper limit of the value of $R$ is restricted to the radius of the interaction cavity and the concern of unwanted mode transformation, then $R$ is designed as $1.4 \mathrm{~mm}$, at which $\theta_{\mathrm{BLF}}$ and $\theta_{\mathrm{BHF}}$ are determined by (1) as $58.3^{\circ}$ and $52.8^{\circ}$, respectively.

On the other hand, the helical cut of Vlasov launcher should cover the Brillouin regions of both modes. For the $T E_{1,3}$ mode, the Brillouin region can be explicitly defined by the axial length $L_{\mathrm{LF}}$, the azimuthal length $L_{\mathrm{aLF}}$, and the inclination angle $\Phi_{\mathrm{LF}}$. The Brillouin region for $T E_{4,9}$ mode can also be determined in a similar fashion. These values as listed in Table II are determined by (3), (4), and (6).
TABLE II

Dimensional Parameters of the Brillouin Regions of the Two Modes

\begin{tabular}{lccccc}
\hline \hline$L_{\mathrm{LF}}$ & $L_{\mathrm{HF}}$ & $L_{\mathrm{aLF}}$ & $L_{\mathrm{aHF}}$ & $\Phi_{\mathrm{LF}}$ & $\Phi_{\mathrm{HF}}$ \\
\hline $3.70 \mathrm{~mm}$ & $4.59 \mathrm{~mm}$ & $4.07 \mathrm{~mm}$ & $4.05 \mathrm{~mm}$ & $67.2^{\circ}$ & $62.5^{\circ}$ \\
\hline \hline
\end{tabular}

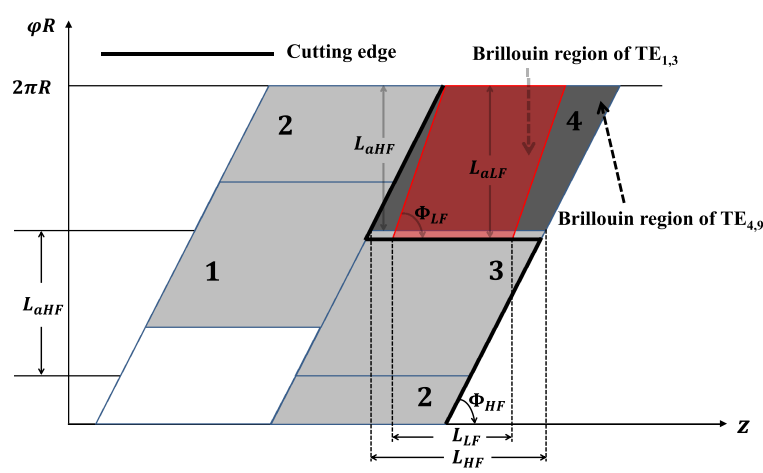

Fig. 4. Unfolded view of the Vlasov launcher and the Brillouin regions of the two modes.

The Brillouin region for the $T E_{1,3}$ mode at $342 \mathrm{GHz}$ is shown in red in Fig. 4. We have also shown four neighboring Brillouin regions (numbered as $1,2,3$, and 4 ) of the $T E_{4,9}$ mode at $1368 \mathrm{GHz}$, the last one of which is in dark gray. It can be seen that $L_{\mathrm{LF}}$ is shorter than $L_{\mathrm{HF}}$, while $L_{\mathrm{aLF}}$ is longer than $L_{\mathrm{aHF}}$. To enclose the last Brillouin regions of both modes, the traditional helical cut of the launcher, instead of being determined by $L$ and $L_{a}$ of single mode, should be modified by considering both modes. That is, the azimuthal and the axial lengths should be chosen as $L_{\mathrm{aLF}}$ and $L_{\mathrm{HF}}$, respectively, and the new cut is shown as the bolded line in Fig. 4. In such a way, the launcher will be capable of launching the two modes effectively.

The radiated rays of the modified Vlasov launcher are parallel in the $y z$ plane and spread an angle of $2 \theta$ in the $x y$ plane. A quasi-parabolic cylinder mirror can be used to parallelize the rays in the $x y$ plane. After the adjustment, the rays are parallel in both $x y$ and $y z$ plane. Then, an elliptic-paraboloid [19] mirror is placed in such a way that its axis is parallel with the propagation direction of the rays to reflect and focus them to the focal point. The transverse view of the synthesis of the DQMC is shown in Fig. 5. The path of the wave beam in the DQMC is represented by the red lines with arrows.

The dimension of the quasi-parabolic cylinder mirror in the $x y$ plane is governed by [20]

$$
\begin{aligned}
& x(\phi)=R_{c}+\pi R_{c} \cot \left(\frac{\phi}{2}\right)-R_{c} \phi \cot \left(\frac{\phi}{2}\right)-2 l_{1} \cot \left(\frac{\phi}{2}\right) \\
& y(\phi)=R_{c} \cot \left(\frac{\phi}{2}\right)+\left(\pi \frac{R_{c}}{2}-R_{c} \frac{\phi}{2}-l_{1}\right)\left(\cot ^{2}\left(\frac{\phi}{2}\right)-1\right)
\end{aligned}
$$

in which $l_{1}$ is the distance between the vertex of the quasiparabolic cylinder mirror and the $z$-axis. $\phi$ represents the angle between the projections of the radiated rays in the $x y$ plane and 


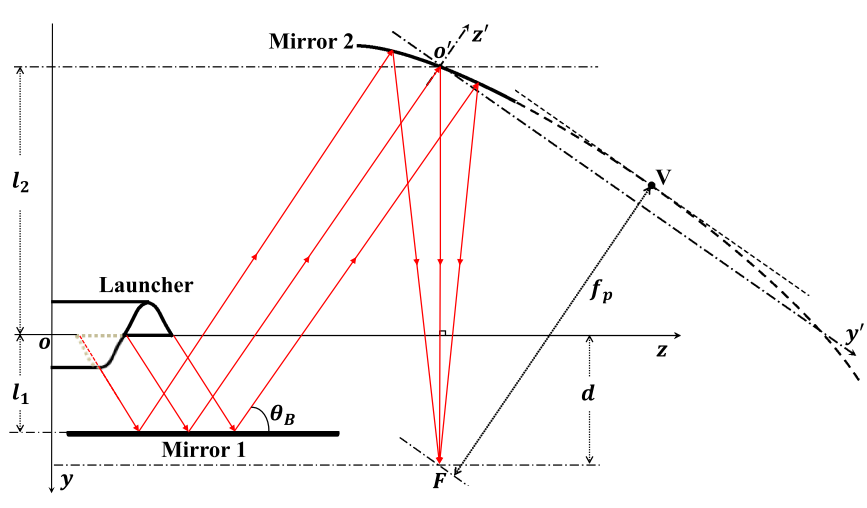

Fig. 5. Side view of the scheme of mirror synthesis.

TABLE III

PARAMETERS FOR THE MIRROR SYNTHESIS

\begin{tabular}{lccccc}
\hline \hline$R_{c}$ & $l_{1}$ & $l_{2}$ & $l_{0}$ & $\theta_{B}$ & $d$ \\
\hline $0.18 \mathrm{~mm}$ & $4.2 \mathrm{~mm}$ & $14.0 \mathrm{~mm}$ & $1.0 \mathrm{~mm}$ & $52.8^{\circ}$ & $22.4 \mathrm{~mm}$ \\
\hline \hline
\end{tabular}

the $y$-axis [20]. The shape of the quasi-parabolic cylinder mirror is consistent along the $z$-direction.

The original point of the local coordinate system for the elliptic-paraboloid mirror is $O^{\prime}$. The distance between $O^{\prime}$ and $O$ is $l_{2}$ in the $y$-direction. In the $\left(x, y^{\prime}, z^{\prime}\right)$ coordinate system, the equation of the elliptic-paraboloid mirror can be written as

$$
z^{\prime}=-\frac{\left(y^{\prime}-l_{F O^{\prime}} \cdot \cos \theta_{B}\right)^{2}}{4 f_{p}}-\frac{\left(x^{\prime}\right)^{2}}{4 f_{p}}+\frac{\left(l_{F O^{\prime}} \cdot \cos \theta_{B}\right)^{2}}{4 f_{p}}
$$

where $l_{F O^{\prime}}$ is the distance between the focusing point $F$ and $O^{\prime}$. By setting $F$ being right below $O^{\prime}$ and having a distance of $d$ from the $z$-axis, the axis $z^{\prime}$ forms an angle of $\theta_{B}$ with the $z$-axis, and

$$
f_{p}=\frac{l_{F O^{\prime}}}{2}\left(1+\sin \theta_{B}\right) .
$$

The chosen values of the variables used in (8)-(10) are listed in Table III. The values of $R_{c}$ and $\theta_{B}$ are calculated according to the parameters of the $T E_{4,9}$ mode at $1368 \mathrm{GHz}$. This is because the two modes share similar values of $R_{c}$ and $\theta_{B}$, and the $T E_{1,3}$ mode at $342 \mathrm{GHz}$ is less sensitive to the parameter variations.

To verify the above design, a 3-D full electromagnetic simulation using Feko 7.0 [21] is conducted. The model is built according to the scheme shown in Fig. 5. A virtual observation window is located in the $y=22.4 \mathrm{~mm}$ plane to record the field distribution of the wave beams at the waists.

The distributions of the total electric field $E$ and the corresponding $x$ component $E_{x}$ on the focal plane, for the cases of $T E_{1,3}$ mode at $342 \mathrm{GHz}$ and $T E_{4,9}$ mode at $1368 \mathrm{GHz}$, are shown in Figs. 6 and 7, respectively. The $y$ components $E_{y}$ and $z$ components $E_{z}$ on the focal plane are below $-25 \mathrm{~dB}$ for the above two cases (not shown). It can be seen that both the highorder modes at the fundamental and the fourth harmonic can be converted into quasi-Gaussian beams, and the side lobes of the converted beams are lower than $-20 \mathrm{~dB}$ at $342 \mathrm{GHz}$ and lower

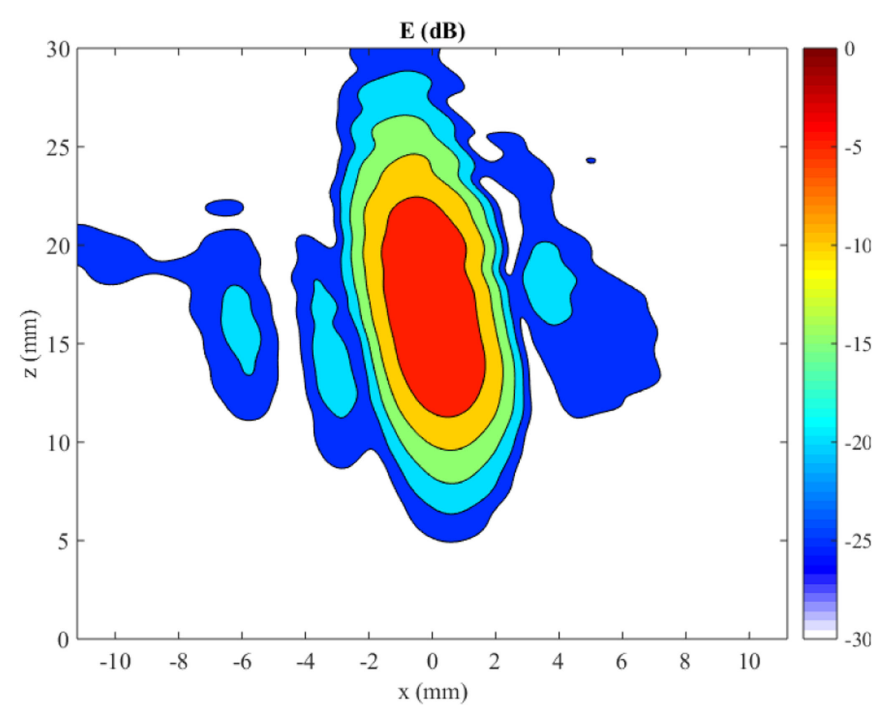

(a)

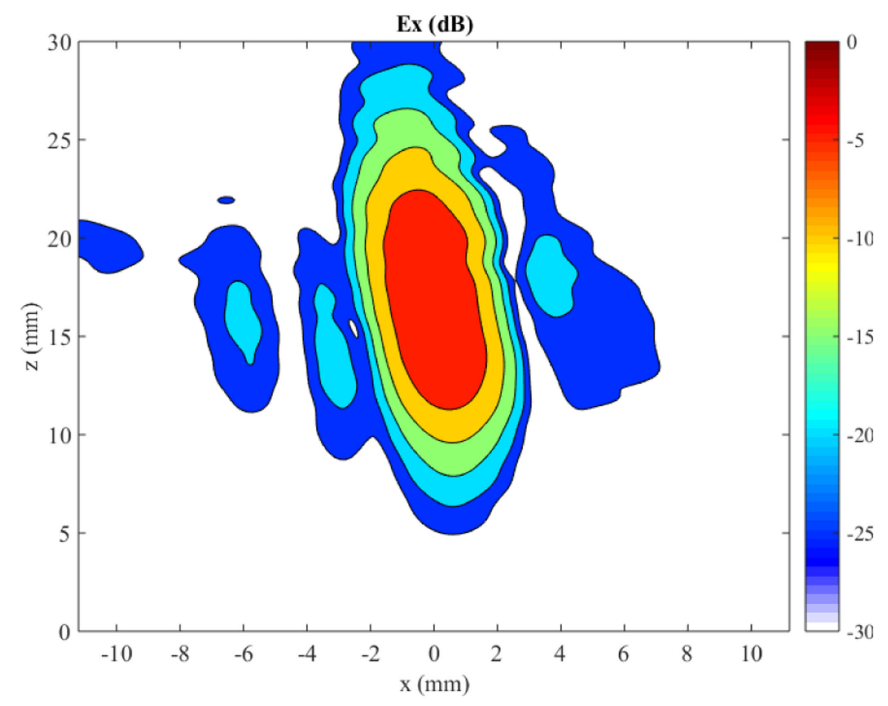

(b)

Fig. 6. Normalized electric field distribution (dB) with respect to the peak electric field strength of $2.25 \times 10^{5} \mathrm{~V} / \mathrm{m}$ on the observation window for $T E_{1,3}$ mode incidence at $342 \mathrm{GHz}$. (a) Total electric field. (b) $E_{x}$ component.

than $-15 \mathrm{~dB}$ at $1368 \mathrm{GHz}$. Figs. 6 and 7 also suggest that both beams are linearly polarized in the $x$-direction.

Table IV summarizes the scalar Gaussian content of the converted wave beam $\left(C_{s}\right)$ [19], the power $P$ being collected on the observation plane [13], the power transmission coefficient $\eta$, and the fraction of depolarized power $(X P)$ [22] for the above quasi-Gaussian beams.

We can conclude here that by using the designed DQMC, both modes at different harmonics can be transformed into quasiGaussian beams, in a similar quality and fashion as in the singlemode QMC [23], [24].

It should be noticed that the similar propagation properties of the two modes are essential to the optimal performance of the designed DQMC. As seen from the mode selection criteria and the operation principle of the gyro-multiplier [4], [5], the $k_{0}, k_{z}$, $k_{\perp}, m, \mu_{m, n}^{\prime}$, and $\omega$ of the $T E_{4,9}$ mode are (nearly or exactly) four times in value of their counterparts in $T E_{1,3}$ mode. This 


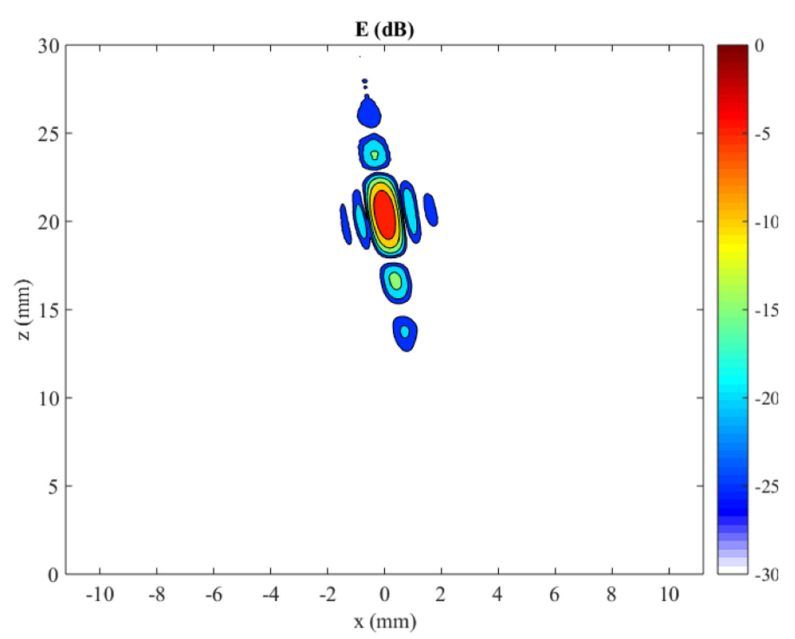

(a)

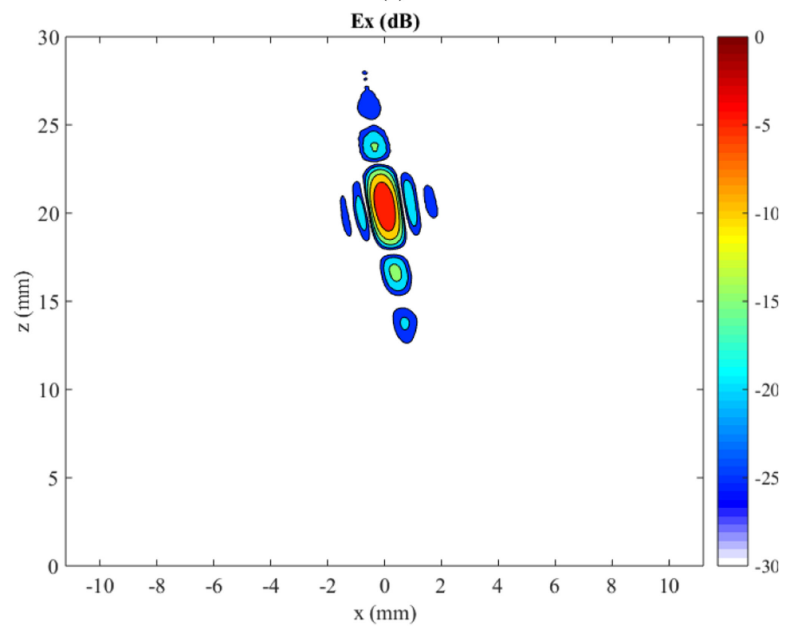

(b)

Fig. 7. Normalized electric field distribution $(\mathrm{dB})$ with respect to the peak electric field strength of $2.38 \times 10^{5} \mathrm{~V} / \mathrm{m}$ on the observation window for $T E_{4,9}$ mode incidence at $1368 \mathrm{GHz}$. (a) Total electric field. (b) $E_{x}$ component.

TABLE IV

Gaussian Contents, the Power Being Collected on the OBSERVATION Plane, Power Transmission COEFFicients, AND THE FraCtions of DEPOLARIZED POWER FOR THE TWO MODES

\begin{tabular}{lcc}
\hline \hline & $342 \mathrm{GHz}$ & $1368 \mathrm{GHz}$ \\
\hline$C_{s}$ & $94.6 \%$ & $89.6 \%$ \\
$P(\mathrm{~kW})$ & 1.628 & 0.109 \\
$\eta\left(P / P_{0}\right)$ & $81.4 \%$ & $90.6 \%$ \\
$X P$ & $0.8 \%$ & $0.8 \%$ \\
\hline
\end{tabular}

in turn leads to similar values of $\theta_{B}, L, L_{a}$, and $\Phi$ for the two modes.

\section{HIGH-POWER THz FSS}

To avoid overheating problem and fabricating very fine structure [25], a freestanding FSS is designed by employing periodic circular perforations on a brass plate, as shown in Fig. 8 . Although this type of FSS has been reported in a number of literatures [26]-[28], its integration with a high-power vacuum electronic device and operation with oblique incident wave at

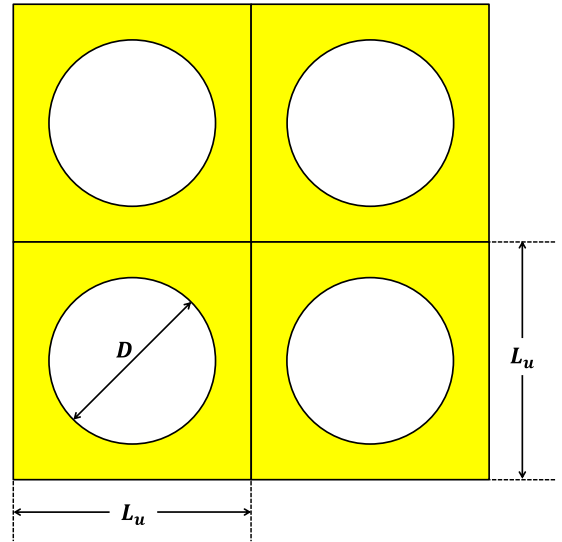

Fig. 8. Sizes of the unit cell for the designed FSS.

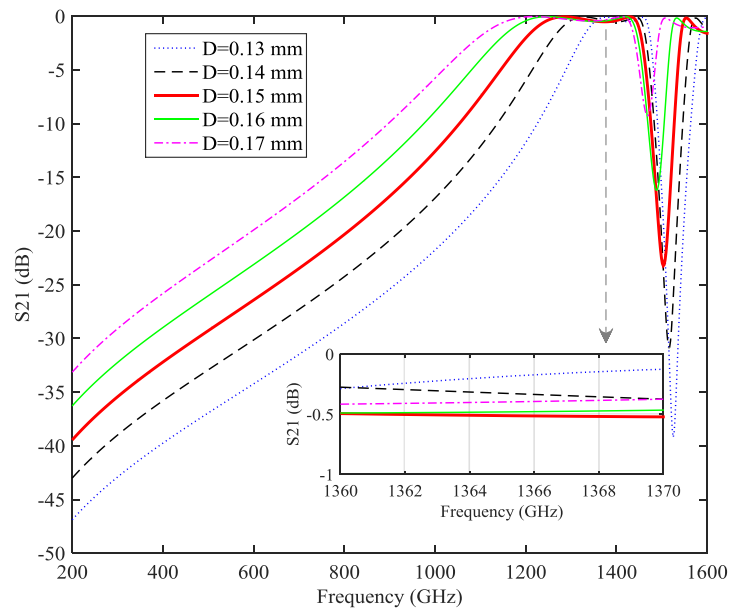

(a)

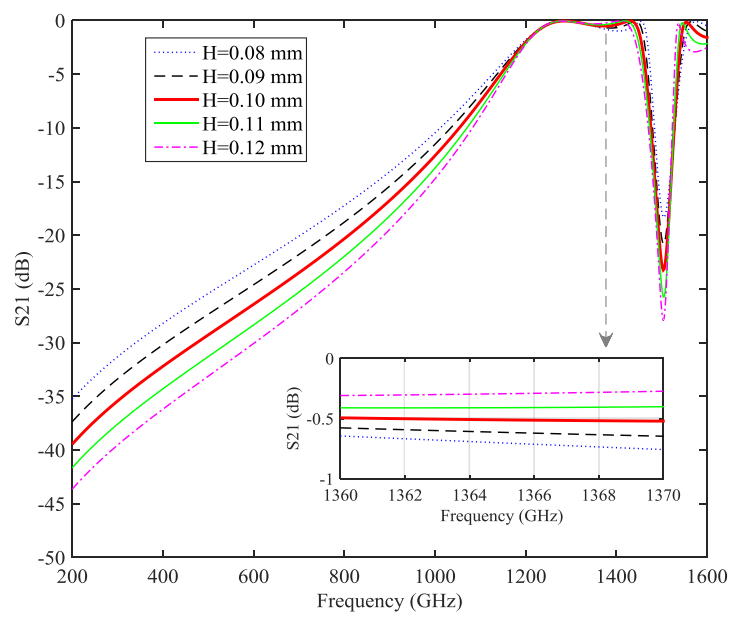

(b)

Fig. 9. $S_{21}$ parameters of the designed FSS and the sensitivity test regarding $D$ and $H$ with CST Microwave Studio. (a) Sensitivity test regarding $D$. (b) Sensitivity test regarding $H$.

frequencies over $1 \mathrm{THz}$ have not been studied elsewhere. The lengths of the square unit cell $L_{u}$ are $190 \mu \mathrm{m}$. The diameter of the circular perforation $D$ is $150 \mu \mathrm{m}$, and the thickness $H$ of the brass plate is $100 \mu \mathrm{m}$. The designed angle of incidence (as $\theta_{\text {in }}$ in Fig. 1) for the FSS is $15^{\circ}$. 


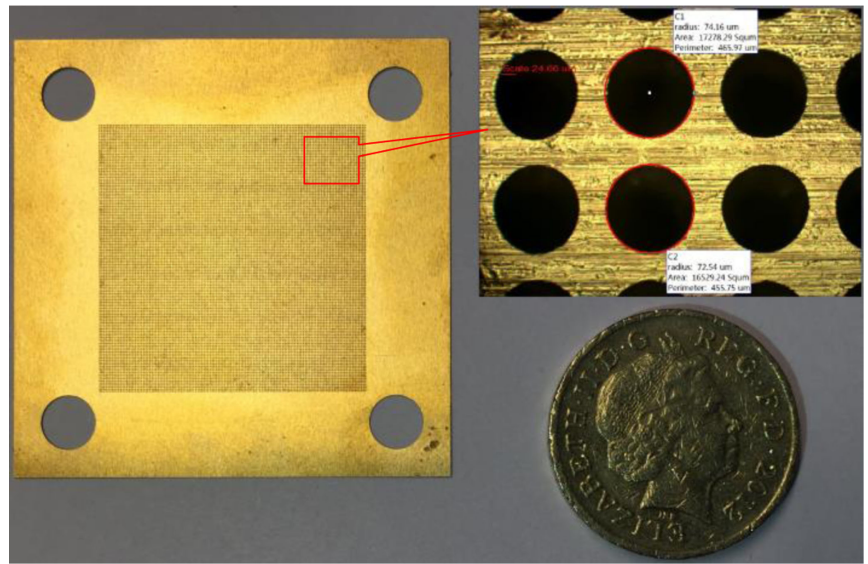

Fig. 10. Fabricated FSS sample and the regional microscopic image.

TABLE V

COMPARISON BETWEEN THE DESIGNED AND AVERAGED FABRICATED PARAMETERS

\begin{tabular}{lccc}
\hline \hline & $L_{u}(\mu \mathrm{m})$ & $D(\mu \mathrm{m})$ & $H(\mu \mathrm{m})$ \\
\hline Designed & 190 & 150 & 100 \\
Fabricated & 187 & 149 & 97 \\
\hline \hline
\end{tabular}

The above design of FSS is modeled in CST Microwave Studio [29] to evaluate the performance. The conductivity of brass is chosen as $0.698 \times 10^{7} \mathrm{~S} / \mathrm{m}$ in the simulation. The $S_{21}$ parameter of the designed FSS is shown in Fig. 9(a) by the bolded red curve. The simulation result has shown an $S_{21}$ value of $-34.01 \mathrm{~dB}$ at $342 \mathrm{GHz}$ and that of $-0.52 \mathrm{~dB}$ at $1368 \mathrm{GHz}$, indicating that the low-frequency content will be reflected while the high-frequency counterpart will be transmitted. Thus, the two frequencies can be separated by the designed FSS with good isolation. Fig. 9(a) also shows that as $D$ varies from 130 to $170 \mu \mathrm{m}$, the insertion loss at $342 \mathrm{GHz}$ remains higher than $27.66 \mathrm{~dB}$, while that at $1368 \mathrm{GHz}$ stays below $0.52 \mathrm{~dB}$. Fig. 9(b) shows that as $H$ varies from 80 to $120 \mu \mathrm{m}$, the insertion loss at $342 \mathrm{GHz}$ remains higher than $29.94 \mathrm{~dB}$, while that at $1368 \mathrm{GHz}$ keeps lower than $0.74 \mathrm{~dB}$. Thus, the designed FSS can tolerate a fabrication error of $20 \mu \mathrm{m}$ regarding $D$ and $H$, and the insertion loss can be maintained as below $1 \mathrm{~dB}$ given the above fabrication error. Such accuracy can be provided by the etching technology.

The etching fabrication process of the FSS can be divided into four steps: surface polishing, protective film coating, chemical corrosion, and postprocessing. Before the experimental test, the FSS surface is immersed in distilled water and cleaned by ultrasound. Fig. 10 shows the etched FSS sample, which is formed by an array of $105 \times 105$ circular perforations. A regional microscopic image of the fabricated FSS is shown in the inset. Good alignment of the holes can be observed. The comparison between the designed parameters and the averaged fabricated sizes is shown in Table V. The diameters of the individual perforations vary from 144 to $152 \mu \mathrm{m}$, which are within the range of sensitivity test. The root-mean-squared (rms) surface roughness is measured as $399 \mathrm{~nm}$ by a DektakXT stylus surface profiler [30]. By taking this rms value into the HammerstadBekkadal formula [31], [32], the effective conductivity of brass

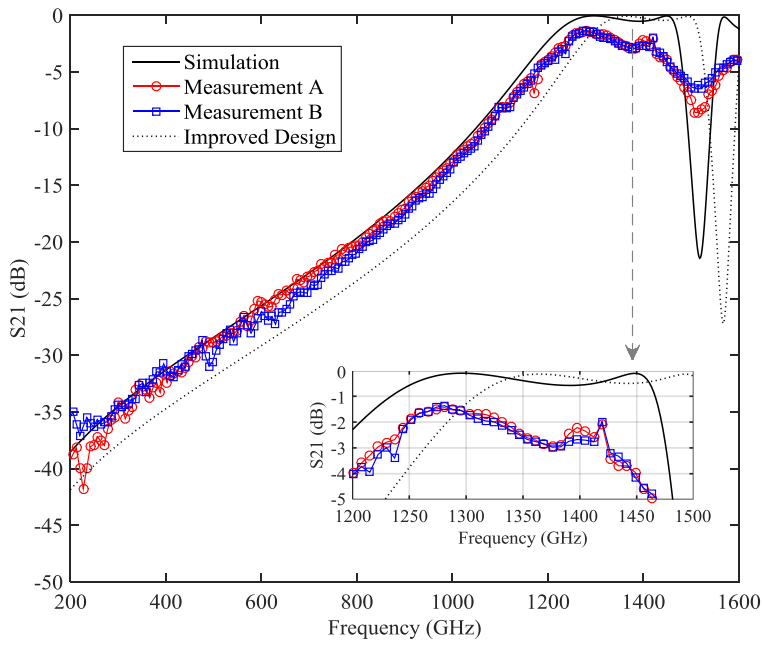

Fig. 11. Comparison between the simulation and test results. The black curve is the simulated $S_{21}$ performance with the measured sizes listed in Table V. The red circles and blue squares are the measured $S_{21}$ values when the two sides of the FSS sample are facing the incoming testing beam, respectively. The dotted curve shows the transmission property of the proposed improved design.

is estimated as $0.741 \times 10^{7}$ and $0.698 \times 10^{7} \mathrm{~S} / \mathrm{m}$ at 342 and $1368 \mathrm{GHz}$, respectively. Since the effective conductivity variation with frequency is small and has negligible impact on the simulated $S_{21}$ performance, its value used in the previous and the following simulations is chosen as $0.698 \times 10^{7} \mathrm{~S} / \mathrm{m}$.

The designed FSS is then tested in a THz-TDS system [33]. Fig. 11 shows the comparison between the simulated and the measured $S_{21}$ curves. The tested insertion loss at $342 \mathrm{GHz}$ is $32.7 \mathrm{~dB}$, showing good agreement with the simulation result. The simulated and measured $S_{21}$ agree well with each other in the frequency range from 0.2 to $1 \mathrm{THz}$, while the divergence grows beyond $1 \mathrm{THz}$. This is not unexpected since the transmission of FSS becomes more and more sensitive to the fabrication errors, such as roughness and flatness of the slab, variation of hole size, and slab thickness, as the frequency increases. These factors also explain the relatively higher insertion loss of $2.82 \mathrm{~dB}$ than the designed value of $0.5 \mathrm{~dB}$ at $1368 \mathrm{GHz}$. Last but not the least, the lowest value of the insertion loss is $1.33 \mathrm{~dB}$ at about $1281 \mathrm{GHz}$ (transmission peak), which is about $100 \mathrm{GHz}$ higher than the cutoff frequency of the circular perforations.

The above analysis naturally leads to two possible methods to improve the FSS performance at $1368 \mathrm{GHz}$. One method is to employ a better fabrication technique other than the etching process, such as the computer numerically controlled milling machining, together with gold plating. The other method is to reduce $L_{u}$ and $D$ to 0.18 and $0.14 \mathrm{~mm}$, respectively, which will raise the cutoff frequency of the perforations. By doing so, the transmission peak goes up in the frequency range around $1368 \mathrm{GHz}$, instead of $1281 \mathrm{GHz}$, as shown by the dotted curve in Fig. 11.

In addition, the power handling capability of the designed FSS should be paid special attention to. Since the gyro-multiplier is to be operated with pulsed power supply, the temperature rise of the FSS is estimated to be lower than $50{ }^{\circ} \mathrm{C}$, which will not cause overheating problem to the metal slab made of brass. 
For the electric breakdown, we need to consider a simultaneous incidence of two plane waves with the electric field strengths of $2.25 \times 10^{5}$ and $2.38 \times 10^{5} \mathrm{~V} / \mathrm{m}$, respectively. These two values are the maximum electric field strengths of the generated quasi-Gaussian beams at 342 and $1368 \mathrm{GHz}$, respectively. The maximum electric field on the FSS can be estimated by calculating the maximum field enhancement factor (MFEF) [10]. The values of the MFEF can be obtained from the full-wave EM simulation with CST Microwave Studio as 1.19 at $342 \mathrm{GHz}$ and 3.28 at $1368 \mathrm{GHz}$, respectively. Therefore, the values of the maximum electric fields induced on the FSS by the two plane waves are $0.27 \times 10^{6}$ and $0.78 \times 10^{6} \mathrm{~V} / \mathrm{m}$, respectively. The maximum total electric field strength of $1.05 \times 10^{6} \mathrm{~V} / \mathrm{m}$ is lower than the breakdown electric field strength of the air $E_{a}=3 \times 10^{6} \mathrm{~V} / \mathrm{m}$. Hence, the designed FSS is capable of handling the high-power $\mathrm{THz}$ output from this gyro-multiplier. For other cases where much higher output power levels are involved, the methods of breakdown prevention by using nonresonant elements [10] or layered structures [34] in the FSSs can be considered.

\section{CONCLUSION}

A challenging output system for the gyro-multiplier with sectioned cavity is designed based on the combination of a DQMC and a high-power FSS. The performance of the DQMC is shown to be optimal due to the fact that the two specially selected modes of the gyro-multiplier exhibit similar propagation properties. The 3-D full-wave simulation has verified good linearity, low cross polarization, and high Gaussian content in the converted quasi-Gaussian beams. At the same time, a high-pass FSS is shown to separate well the two output waves by their frequencies in simulation and measurement. Also, the theoretical analysis has shown that the designed FSS can cope with the high-power radiation of the quasi-Gaussian beams.

It is anticipated that the design method devised in this paper can be applied to other gyro-devices with frequency multiplication, for example, the gyro-multiplier with corrugated cavity [5].

\section{ACKNOWLEDGMENT}

The authors would like to thank X. Cao, Beijing University of Posts and Telecommunications, for his assistance during the fabrication process of the FSS, and Y. Zeng, Dr. O. Sushko, Dr. M. Candotti, Dr. M. Torrico, and Dr. R. Donnan, all with Queen Mary University of London, for their suggestions in the measurement of the FSS.

\section{REFERENCES}

[1] M. Y. Glyavin, T. Idehara, and S. P. Sabchevski, "Development of THz gyrotrons at IAP RAS and FIR UF and their applications in physical research and high-power THz technologies," IEEE Trans. THz Sci. Technol., vol. 5, no. 5, pp. 788-797, Sep. 2015.

[2] I. Bandurkin and S. Mishakin, "Gyromultiplier with sectioned cavity," Phys. Plasmas, vol. 17, 2010, Art. no. 110706.

[3] I. Bandurkin, V. Bratman, and A. Savilov, "Frequency multiplication in gyrotron autooscillators," Tech. Phys. Lett., vol. 32, pp. 84-87, 2006.

[4] A. Savilov and G. Nusinovich, "On the theory of frequency-quadrupling gyroklystrons," Phys. Plasmas, vol. 14, 2007, Art. no. 053113.
[5] I. Bandurkin, V. Bratman, A. Savilov, S. Samsonov, and A. Volkov, "Experimental study of a fourth-harmonic gyromultiplier," Phys. Plasmas, vol. 16, 2009, Art. no. 070701.

[6] D. A. Constable, "The numerical and experimental investigation of gyromultiplier configurations," Ph.D. dissertation, Dept. Phys., Univ. Strathclyde, Glasgow, Scotland, U.K., 2013.

[7] S. N. Vlasov and I. Orlova, "Quasioptical transformer which transforms the waves in a waveguide having a circular cross section into a highly directional wave beam," Radiophys. Quantum Electron., vol. 17, pp. 115-119, 1974.

[8] O. Prinz et al., "Highly efficient quasi-optical mode converter for a multifrequency high-power gyrotron," IEEE Trans. Electron Devices, vol. 56, no. 5, pp. 828-834, May 2009.

[9] Y. Wang, B. Yang, Y. Tian, R. S. Donnan, and M. J. Lancaster, "Micromachined thick mesh filters for millimeter-wave and terahertz applications," IEEE Trans. THz Sci. Technol., vol. 4, no. 2, pp. 247-253, Mar. 2014.

[10] M. Li and N. Behdad, "Frequency selective surfaces for pulsed high-power microwave applications," IEEE Trans. Antennas Propag., vol. 61, no. 2, pp. 677-687, Feb. 2013

[11] T. Idehara, T. Tatsukawa, G. Brand, P. Fekete, and K. Moore, "A twodimensionally focusing, quasi-optical antenna for millimeter-wave scattering in plasmas," J. Appl. Phys., vol. 67, pp. 7086-7091, 1990.

[12] J. Lorbeck and R. Vernon, "Dual-shaped dual-reflector antenna for a whispering-gallery-mode gyrotron," in Proc. Antennas Propag. Soc. Int. Symp., London, ON, Canada, 1991, pp. 452-455.

[13] J. Jin, "Quasi-optical mode converter for a coaxial cavity gyrotron," Ph.D. dissertation, Inst. Pulsed Power Microw. Technol., Karlsruhe Inst. Technol., Karlsruhe, Baden-Württemberg, Germany, 2007.

[14] G. Denisov et al., "110 GHz gyrotron with a built-in high-efficiency converter," Int. J. Electron., vol. 72, pp. 1079-1091, 1992.

[15] G. Denisov, A. Bogdashov, I. Gachev, S. Mishakin, and S. Samsonov, "New radiation input/output systems for millimeter-wave gyrotron traveling-wave tubes," Radiophys. Quantum Electron., vol. 58, pp. 769-776, 2016.

[16] A. Chirkov et al., "Use of Huygens' principle for analysis and synthesis of the fields in oversized waveguides," Radiophys. Quantum Electron., vol. 49, pp. 344-353, 2006.

[17] J. Jin et al., "Novel numerical method for the analysis and synthesis of the fields in highly oversized waveguide mode converters," IEEE Trans. Microw. Theory Techn., vol. 57, no. 7, pp. 1661-1668, Jul. 2009.

[18] J. M. Neilson, "Optimal synthesis of quasi-optical launchers for highpower gyrotrons," IEEE Trans. Plasma Sci., vol. 34, no. 3, pp. 635-641, Jun. 2006.

[19] J. H. Flamm, "Diffraction and scattering in launchers of quasi-optical mode converters for gyrotrons," Ph.D. dissertation, Inst. Pulsed Power Microw. Technol., Karlsruhe Inst. Technol., Karlsruhe, Baden-Württemberg, Germany, 2012.

[20] C. J. Edgcombe, Gyrotron Oscillators: Their Principles and Practice. London, U.K.: Taylor \& Francis, 1993.

[21] FEKO User Help Document, Altair Corp., Lincolnshire, IL, USA (2015). [Online]. Available: https://www.feko.info/

[22] J.-P. Hogge, T. Tran, P. Paris, and M. Tran, "Operation of a quasioptical gyrotron with a Gaussian output coupler," Phys. Plasmas, vol. 3, pp. 3492-3500, 1996.

[23] M. Blank, "High efficiency quasi-optical mode converters for overmoded gyrotrons," Ph.D. dissertation, Dept. Elect. Eng. Comput. Sci., Massachusetts Inst. Technol., Cambridge, MassachusettsMA, USA, 1994.

[24] B. Rock and A. W. Fliflet, "Analysis and design of a quasi-optical mode converter for a 1-kW, 550-GHz, TE15,2-mode gyrotron," IEEE Trans. THz Sci. Technol., vol. 3, no. 5, pp. 641-648, Sep. 2013.

[25] B. A. Munk, Frequency Selective Surfaces: Theory and Design. New York, NY, USA: Wiley, 2000.

[26] C.-C. Chen, "Transmission of microwave through perforated flat plates of finite thickness," IEEE Trans. Microw. Theory Techn., vol. 21, no. 1, pp. 1-6, Jan. 1973.

[27] F. Miyamaru and M. Hangyo, "Finite size effect of transmission property for metal hole arrays in subterahertz region," Appl. Phys. Lett., vol. 84, pp. 2742-2744, 2004.

[28] C. Winnewisser, F. T. Lewen, M. Schall, M. Walther, and H. Helm, "Characterization and application of dichroic filters in the 0.1-3-THz region," IEEE Trans. Microw. Theory Techn., vol. 48, no. 4, pp. 744-749, Apr. 2000.

[29] CST Studio Suite Help Document, CST Corp., Houston, TX, USA. (2015). [Online]. Available: https://www.cst.com/ 
[30] DektakXT Brochure-Bruker, Bruker Corp., Billerica, MA, USA. (2015) [Online]. Available: https://www.bruker.com

[31] S. Lucyszyn, "Investigation of anomalous room temperature conduction losses in normal metals at terahertz frequencies," Proc. Inst. Elect. Eng.Microw., Antennas, Propag., vol. 151, no. 4, pp. 321-329, Aug. 2004.

[32] M. Kirley and J. H. Booske, "Terahertz conductivity of copper surfaces," IEEE Trans. THz Sci. Technol., vol. 5, no. 6, pp. 1012-1020, Nov. 2015.

[33] A. I. McIntosh, B. Yang, S. M. Goldup, M. Watkinson, and R. S. Donnan, "Terahertz spectroscopy: A powerful new tool for the chemical sciences?" Chem. Soc. Rev., vol. 41, pp. 2072-2082, 2012.

[34] C.-H. Liu and N. Behdad, "High-power microwave filters and frequency selective surfaces exploiting electromagnetic wave tunneling through negative layers," J. Appl. Phys., vol. 113, 2013, Art. no. 064909.

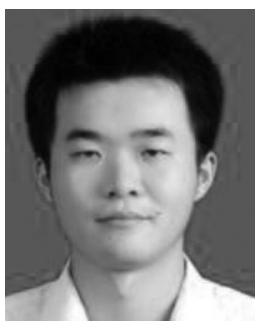

Xiang $\mathbf{L i}$ (S'13) received the B.S. degree in vacuum electronics technology from the University of Electronic Science and Technology of China, Chengdu, China, in 2009. He is currently working toward the $\mathrm{Ph} . \mathrm{D}$. degree in electronic engineering at the School of Electronic Engineering and Computer Science, Queen Mary University of London, London, U.K.

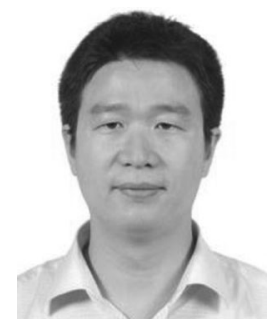

Xiaoming Liu received the B.Sc. degree in applied physics from the Nanjing University of Posts and Telecommunications, Nanjing, China, in 2006, and the Ph.D. degree in electronic engineering from the School of Electronic Engineering and Computer Science, Queen Mary University of London, London, U.K. in 2012 .

In 2012, he joined the School of Electronic Engineering, Beijing University of Posts and Telecommunications, Beijing, China. His research interests include terahertz science and technology, quasi-optical techniques and systems, millimeter and submillimeter wave antenna measurement techniques, and bioelectromagnetics.

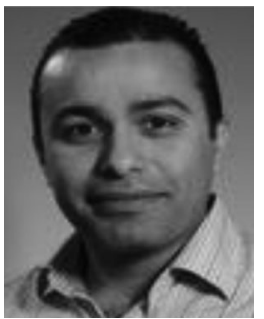

Yasir Alfadhl (M'09) was born in Najaf, Iraq, in 1978. He received the B.S. degree in telecommunication engineering from the Queen Mary University of London, London, U.K., in 2000, and the Ph.D. degree from the University of London, London, U.K., in the areas of electromagnetics and antennas in telecommunication engineering in 2006 .

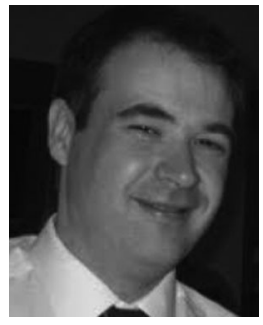

Kevin Ronald was born in Glasgow, U.K. He received the B.Sc. (with honors) and Ph.D. degrees in physics from the University of Strathclyde, Glasgow, U.K., in 1992 and 1997, respectively.

He is currently a Lecturer with the Scottish Universities Physics Alliance, Department of Physics, University of Strathclyde, Glasgow, U.K.

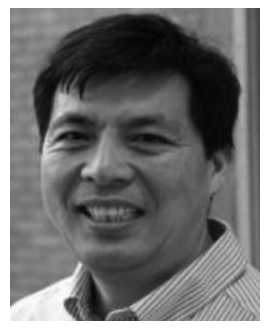

Wenlong He received the B.Sc. degree in physics from Suchow University, Suchow, Jiangsu, China, in 1983, the M.Sc. degree in accelerator physics from the China Academy of Engineering Physics, Chengdu, China, in 1988, and the Ph.D. degree in relativistic electron beams and masers from the Department of Physics, University of Strathclyde, Glasgow, U.K., in 1995.

$\mathrm{He}$ is currently an Advanced Fellow at the Scottish Universities Physics Alliance, Department of Physics, University of Strathclyde. His main research interests include relativistic electron beams, cyclotron autoresonance masers, free-electron lasers, gyro-TWT/BWOs, and other high power microwave devices

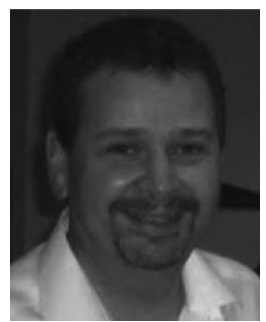

Adrian Cross received the B.Sc. degree (with honors) in physics and the Ph.D. degree from the University of Strathclyde, Glasgow, U.K., in 1989 and 1993, respectively.

He joined the Atoms, Beams, and Plasmas Group, University of Strathclyde, in 1993, initially as a Research Fellow and then as a Lecturer in 2000, a Senior Lecturer in 2005, and currently as a Professor with the Department of Physics. From 2002 to 2007, he was an Engineering and Physical Science Advanced Fellow. He has been involved in various aspects of research on gyrotrons, cyclotron autoresonance masers, free-electron lasers, superradiant sources, gyrotron travelling wave amplifiers, and plasma applications. More recently, he has primarily been concerned with research on microwave pulse compression, $\mathrm{THz}$ radiation sources, and pseudospark physics.

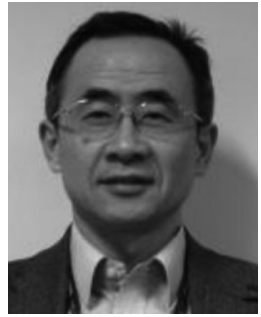

Xiaodong Chen (M'96-SM'07-F'14) is a Professor of microwave engineering with the School of Electronic Engineering and Computer Science, Queen Mary University of London, London, U.K. He is the Director of Joint Research Laboratory on Electromagnetic Theory and Applications between Queen Mary University of London and the Beijing University of Posts and Telecommunications. His research interests include microwave and $\mathrm{THz}$ antennas and devices, wireless communications, and bioelectromagnetics. He has coauthored two textbooks, more than 100 journal papers, and 300 refereed conference papers. 